\title{
Simultaneously Iterative Fast Projection Matching (SI-Faproma)
}

\section{Chun-Chieh Wang $^{1 *}$}

1. National Synchrotron Radiation Research Center, Hsinchu, Taiwan.

* Corresponding author, wang.jay@nsrrc.org.tw

$\mathrm{X}$-ray nano-tomography is a crucial technique for investigating the three-dimensional, fine internal structure of specimens [1]. However, the precise registration of raw projections is difficult to achieve, even when using a highly precise mechanical stage, due to the demanding structural details of specimens at the nanometer level that strongly influence reconstruction fidelity.

To date, the most reliable methods of image registration in X-ray and electron nano-computerized tomography still involve placing high-contrast nanoparticles onto or near the targeted sample to serve as reference points. However, extraneous high-contrast nano-particles are not always found at the desired positions.

Over the past few decades, various markerless auto-alignment algorithms have been proposed to solve these problems, such as the cross-correlation [2], feature matching [3], and projection matching methods [4]. Although the computational speed of cross-correlation is rapid, the most common problem is that cross-correlation methods do not consider the real motion relations between objects through a full rotation, which leads to the subsequent accumulation of registration errors in correlated projections. In contrast, the feature matching and projection matching methods consider the real motion relations between objects; however, the computational cost is very high using these methods.

Recently, some methods have been proposed to reduce the computational cost of traditional projection matching methods, such as reducing the traditional projection matching problem into two onedimensional matching problems [5], or applying a joint iterative method to simultaneously reduce the iteration number of iterative reconstructions and the projection matching [6]. Although both methods have been demonstrated to dramatically reduce the computational cost compared with traditional projection matching methods, the computational time may still possible be further reduced.

Here, we propose the utilization of the joint iterative method to further reduce the computational cost of the fast projection matching (Faproma) algorithm [5], which we called it, simultaneously iterative fast projection matching (SI-Faproma). In Faproma, two correction procedures must be processed. The first procedure simultaneously corrects vertical shifts and rotational axis tilts based on the common-line method; the second procedure corrects horizontal shifts of raw projections captured from different azimuth angles based on the reduced projection matching method. In the second procedure, a temporary $2 \mathrm{D}$ reconstruction generated by the iterative reconstruction is used for projection matching. The temporary iterative reconstruction for each projection matching iterations comprises the time performance bottle neck for both the Faproma and traditional projection matching methods. Applying the joint iterative method, temporary reconstructions, and projection matching processes can be simultaneously processed via one iterative procedure that significantly reduces the processing time required by traditional projection matching methods as well as the second correction procedure of the 
Faproma. In Faproma, the temporary reconstruction of each projection matching iterations is processed using a maximum likelihood reconstruction algorithm after 50 iterations, and the processing time is about 69 seconds per iteration. Figure 1 shows the second correction processing time comparison between Faproma and SI-Faproma. The reconstruction quality is calculated based on the intensity gradient of the reconstruction; higher values represent higher reconstruction quality. We can see that image quality converges after nine iterations using Faproma, with a 625.8s calculation time. However, when using SI-Faproma, the calculation time is reduced to 226s after 91 iterations to achieve the same reconstruction quality. Figure 2 shows the reconstruction quality evolution during SI-Faproma iterations. We can see that the reconstruction reaches acceptable visualization quality after 21 iterations. After 61 iterations, the reconstruction quality reaches the converged value when using Faproma (as shown the dashed line in Fig. 1). The total processing time is only 171s.

In conclusion, we applied a joint iteration method to temporally optimize the second correction procedure of Faproma. The performance of our new method significantly reduced the computational cost to a third of that required by Faproma. This approach speeds up the projection-matching-based image registration to within 10 minutes using a single personal computer.

\section{References:}

[1] C.-C. Wang et al, Scientific Reports 5 (2015), p. 15202.

[2] J Frank et al, in "Electron Tomography”, ed. J. Frank, (Springer US, New York), p. 205-213.

[3] S Brandt et al, Journal of Structural Biology 136 (2001), p. 201.

[4] D. Y. Parkinson et al, Journal of Structural Biology 177 (2012), p. 259.

[5] C.-C. Wang et al, Scientific Reports 7 (2017), p. 3691.

[6] D Gürsoy et al, Scientific Reports 7 (2017), p. 11818.

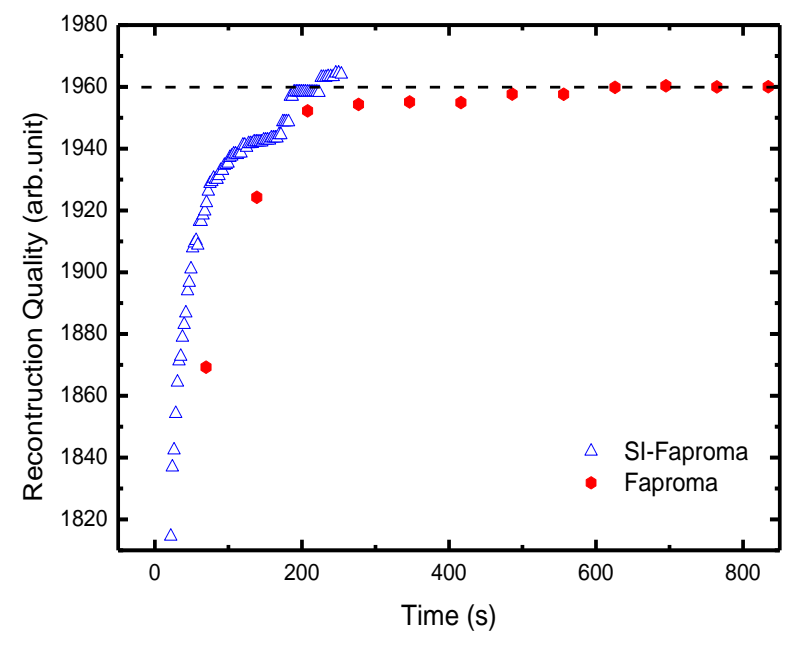

Figure 1. Comparison between Faproma and SI-Faproma for processing performance.

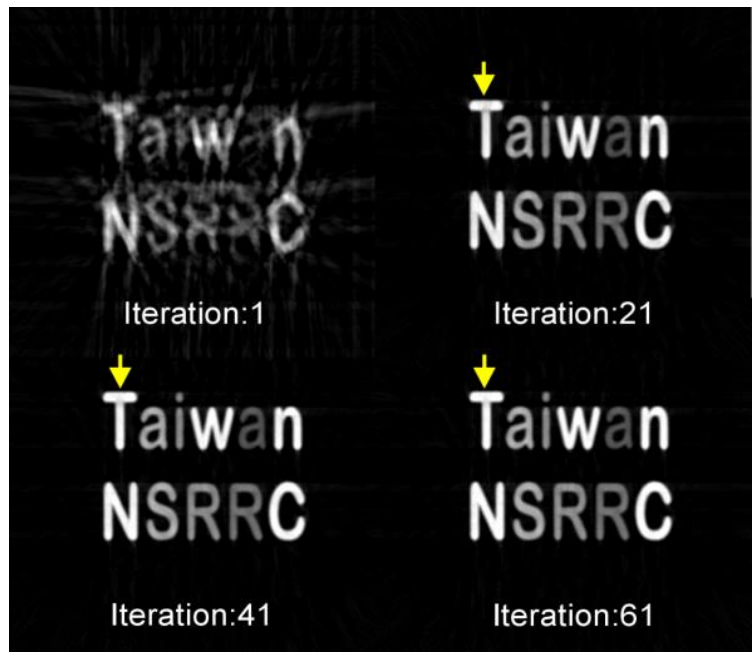

Figure 2. Reconstruction quality evolution during different iteration times using SI-Faproma. The reconstruction reaches acceptable visualization quality after 21 iterations. After 61 iterations, the reconstruction quality reaches the converged value of Faproma. Yellow arrows show very little quality improvement during 21 to 61 iterations. 